\title{
FERRICROMITA : REVISÃO E IMPLICAÇÕES PETROGENÉTICAS
}

\author{
MARIA A.F. CANDIA*, JOSÉ C. GASPAR** E GERGELY A.J. SZABÓ*
}

\begin{abstract}
FERRICHROMITE: REVIEW AND PETROGENETIC IMPLICATIONS Ferrichromite is described in literature as a phase developed over chromite. The most common textural pattern is that of composed described in literature as a phase developed over chromite. The most common textural pattern is that of composed
grains, with a rounded, sharply bounded core mantled by ferrichromite, which is in turn mantled by magnetite, with a gradational interface between them. The formation process for this array is ascribed to serpentinization, through solid-state diffusion of $\mathrm{Mg}, \mathrm{Al}$ and, frequently, $\mathrm{Cr}$, from the oxide-phase towards the surrounding silicates (serpentines, chlorites), and simultaneous entry of Fe. The chemical compositions of the chromite cores may have been modified during this process. Ferrichromite is a submicroscopic intergrowth of oxide phases $\left(\mathrm{R}_{3} \mathrm{O}_{4}, \mathrm{RO}\right.$ e $\left.\mathrm{R}_{2} \mathrm{O}_{3}\right)$, and its chemical composition varies between those of chromite and magnetite, and does not represent a mineral species.
\end{abstract}

Keywords: ferrichromite, serpentinite, composition, genesis.

RESUMO Ferricromita é descçita na literatura como uma fase que se desenvolve às custas de cromita. O padrão textural mais comum corresponde a grãos compostos, com um núcleo de cromita, de contornos nítidos e arredondados, envolto por ferricromita, que, por sua vez, é manteada por magnetita, com contato gradacional. O processo de formação deste arranjo é vinculado à serpeniinização, devido à difusão, em estado sólido, de $\mathrm{Mg}, \mathrm{Al}$ e, freqüentemente, $\mathrm{Cr}$, da fase oxido para os silicatos (serpentinas, clorita) circundantes, e à entrada concomitante de Fe. As composições químicas dos núcleos de cromita podem eventualmente ter sido modificadas por este processo. A ferricromita é constituída de um agregado submicroscópico de fases oxido $\left(\mathrm{R}_{3} \mathrm{O}_{4}, \mathrm{RO}\right.$ e $\left.\mathrm{R}_{2} \mathrm{O}_{3}\right)$, cuja composição química varia entre cromita e magnetita, e não corresponde a uma espécie mineral.

Palavras-chave: ferricromita, serpentinito, composição, gênese.

INTRODUÇÃO A ocorrência de grãos de cromita envolvidos por ferricromita (ferritchromit como definido por Spangenberg 1943) e magnetita é comumente descrita em serpentinitos de derivação ultramáfica. A maioria das ocorrências está em serpentinitos derivados de corpos ultramáficos ofiolíticos (e.g. Onyeagocha 1974, Paraskevopoulos \& Economou 1980, Takla 1982, Michailidis 1995), de porções ultramáficas de complexos estratiformes (Beeson \& Jackson 1969, Nilson 1981) e de komatiitos (Blais \& Auvray 1990, Mitra et al. 1992, Liipo et al. 1995). A ferricromita apresenta ampla variação composicional e não constitui uma espécie mineral em si. Apesar das características químicas do processo de transformação da cromita em ferricromita serem semelhantes em praticamente todas as ocorrências descritas na literatura consultada, a sua gênese é ainda controversa. É feita aqui uma revisão das ocorrências descritas bem como uma avaliação crítica das hipóteses genéticas e suas implicações petrogenéticas. O presente trabalho é parte de um projeto mais amplo que vem sendo desenvolvido pelos autores (Candia 1983, Candia \& Gaspar 1996a, 1996b, 1997, Szabó 1996), visando uma compreensão mais aprofundada dos processos subsolidus que envolvem espinélios cromíferos.

RELAÇÕES TEXTURAIS Caracteristicamente, a associação em grãos compostos de cromita-ferricromita-magnetita é descrita em serpentinitos, constituídos por lizardita/crisotila e/ou antigorita, e quantidades variáveis de clorita. A feição textural mais comum da associação mostra um arranjo concêntrico com um núcleo de cromita circundado por ferricromita, sendo esta envolvida por uma borda externa de magnetita. A interface entre a cromita e a ferricromita é normalmente brusca enquanto que o contato entre a ferricromita e a magnetita é gradacional. A proporção volumétrica entre as três porções é muito variável. O desenvolvimento de ferricromita em fraturas de grãos de cromita é também comumente citado. São também descritas porções irregulares (patches) (Khudeir et al 1992, Mitra et al. 1992)e lamelas de ferricromita (Golding e Bayliss 1968).

A forma dos grãos compostos pelas três fases pode variar em função de fatores tais como (a) hábito da cromita antes do reequilíbrio; (b) dissolução da magnetita da borda externa; (c) sobrecrescimento de magnetita.
Há ocorrências onde cristais euédricos de cromita transformam-se em ferricromita e magnetita, preservando integralmente o hábito magmático (Fig. IA). Nestes casos, há uma clara indicação de que a transformação se processou com conservação do volume original (Beeson \& Jackson 1969, Pinsent \& Hirst 1977, Michailidis 1995). Em outros casos a borda externa de magnetita apresenta golfos e feições de dissolução (Figs. 1B e 1 C) (Michailidis 1995). A identificação de sobrecrescimento de magnetita torna-se mais fácil quando os contornos do cristal de cromita primária podem ainda ser identificados (Fig. 1D) (Ulmer 1974, Bliss \& MacLean 1975, Michailidis 1995). No caso de sobrecrescimentos, podem ocorrer linhas de inclusões (sulfetos, por exemplo) marcando o limite entre a borda de ferricromita e da magnetita sobrecrescida. A figura $1 \mathrm{C}$ mostra, em detalhe, uma ocorrência em que existem duas porções distintas entre a cromita e a magnetita. Ambas podem ser classificadas como ferricromita, mas são composicionalmente diferentes.

O grau de transformação da cromita é variável, desde incipiente até o ponto onde somente resquícios desta fase podem ser identificados. Este é o caso das lentes de magnetita que ocorrem na Grécia (Paraskevopoulos \& Economou 1980), que foram originadas pela transformação quase total de segregações de cromitas.

COMPOSIÇÃO QUÍMICA A natureza da variação composicional entre cromita (núcleo), ferricromita (borda intermediária) e magnetita (borda externa) foi interpretada inicialmente com base nas propriedades ópticas destas fases (Spangenberg 1943). De modo geral, os dados químicos obtidos através de análises químicas pontuais (microssonda eletrônica), mostram concordância com os dados ópticos. Os resultados obtidos por diversos autores (Beeson \& Jackson 1969, Onyeagocha 1974, Ulmer 1974, Bliss \& MacLean 1975, Haggerty 1976, Hoffman \& Walker 1978, Paraskevopoulos \& Economou 1980, Nilson 1981, Takla 1982, Mitra et al. 1992, Michailidis 1990 e 1995, Marques 1996) indicam que a borda de ferricromita, relativamente ao núcleo, mostra nítida diminuição em $\mathrm{Al}_{2} \mathrm{O}_{3}, \mathrm{MgO}$ e, em menor proporção, $\mathrm{Cr}_{2} \mathrm{O}_{3}$, e um concomitante acréscimo em $\mathrm{FeO}$ e $\mathrm{Fe}_{2} \mathrm{O}_{3}$. A borda de magnetita é essencialmente constituída por $\mathrm{FeO}$ e $\mathrm{Fe}_{2} \mathrm{O}_{3}$ (Tabela 1) (Figs. 2 e 3). Exceções podem ocorrer onde o $\mathrm{Cr}_{2} \mathrm{O}_{3}$ apresenta-se constante na 

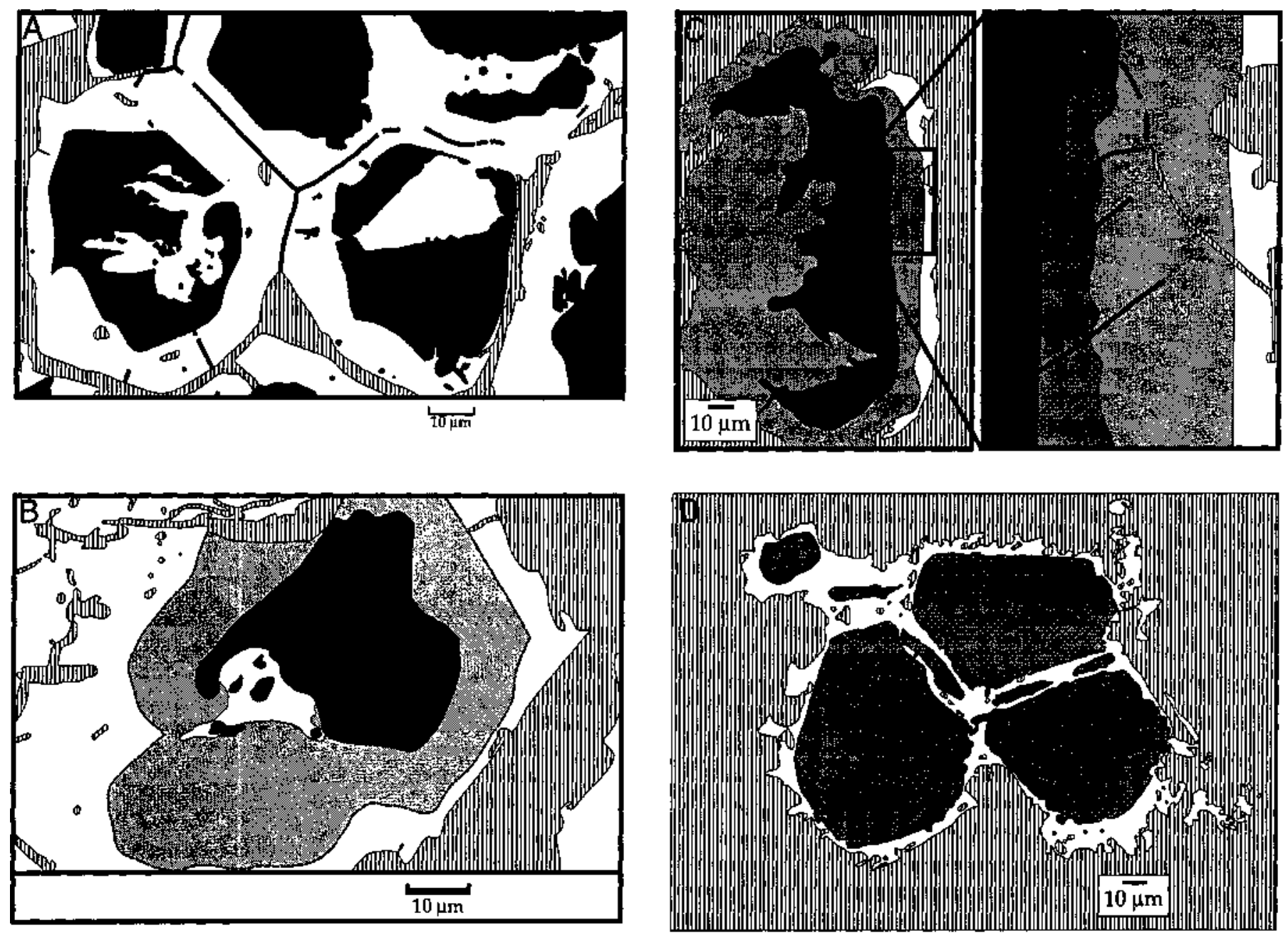

Figura 1 - Texturas de grãos compostos de cromita-ferricromita-magnetita desenhadas a partir de imagens de elétrons retroespalhados extraídas de Michailidis (1995). A - cromitito mostrando textura original após a formação de ferricromita. Preto = cromita e ferricromita; branco = magnetita; hachurado = matriz. B - Grão composto onde é possível observara dissolução de parte da magnetita da borda. Preto = cromita; $\operatorname{cinza}=$ ferricromita; branco = magnetita $;$ hachurado = matriz. $C$ - Grão subédrico mostrando duas zonas distintas de ferricromita (ver detalhe) e dissolução das bordas de magnetita. Preto = cromita; cinza = ferricromita; branco - magnetita; hachurado = matriz. D -Cristais subédricos mostrando sobrecrescimento de magnetita. Preto $=$ cromita e ferricromita $;$ branco $=$ magnetita $;$ hachurado $=$ matriz .

Figure 1 - Textures of chromite-ferrichromite-magnetite composite grains drawn from backscattered electron images from Michailidis (1995). A - preserved original chromitite texture after ferrichromite formation. Black = chromite and ferrichromite; white = magnetite; hatching = matrix. $\mathrm{B}-$ Composite grain showing partial magnetite dissolution. Black = chromite; gray $=$ ferrichromite; white $=$ magnetite; hatching = matrix. $\mathrm{C}-$ Subhedral grain showing two distinct ferrichromite zones (insert) and magnetite dissolution at the rims. Black = chromite; gray = ferrichromite; white = magnetite; hatching = matrix. D - Subhedral crystals with magnetite overgrowth. Black = chromite and ferrichromite; white = magnetite; hatching $=$ matrix.

cromita e na ferricromita (Takla \& Noweir 1980), ou mesmo exibe um aumento na ferricromita (Khudeir et al. 1992). O único caso de trend inverso de variação química no processo de alteração de cromita, citado por Hamlyn (1975), não representa a formação de ferricromita (apesar do autor tê-la assim denominado), mas um reequilíbrio metamórfico da cromita no campo de estabilidade do anfibólio.

Destaca-se nos dados analíticos, um nítido enriquecimento em Ni na borda de magnetita e em manganês na ferricromita (Hoffman \& Walker 1978, Takla 1982, Michailidis 1995) (Figura 2). Outros elementos como Ti, V e Zn ou não mostram variações significativas ou não apresentam padrões constantes entre as várias ocorrências.

Dentro de cada fase, seja cromita, ferricromita ou magnetita, ocorrem variações químicas discretas e contínuas. A variação composicional na ferricromita mostra que, do contato com a cromita em direção à borda de magnetita, há um decréscimo contínuo no componente $\mathrm{MgAl}_{2} \mathrm{O}_{4}$ e enriquecimento em $\mathrm{Fe}_{3} \mathrm{O}_{4}$. Esse mesmo trend pode se estender à magnetita (Figura 2A). No caso dos núcleos de cromita, no entanto, os trends de zonação podem ser incipientes ou não estar presentes (Figura 2B), podem refletir trends originais (relacionados com a sua gênese alpina ou estratiforme) (Figura 2A) ou ainda serem semelhantes aos observados na ferricromita (e.g. Michailides 1995).

Em alguns casos, no domínio da ferricromita, em vez de uma variação química contínua, é descrito um bandamento composicional com bandas alternadas ora ricas ora pobres em $\mathrm{Fe}_{3} \mathrm{O}_{4}$ e um comportamento antipatético para o $\mathrm{Cr}_{2} \mathrm{O}_{3}$ (Figura 



Figura 2 - Perfis químicos de grãos compostos. $C=$ cromita; $F=$ ferrícromita; $M=$ magnetita. $A$ - Extraído de Michailidis (1995). $B$-Extraído de Hoffman \& Walker (1978).

Figure 2 - Chemical profiles of composite grains. $\mathrm{C}=$ chromite; $\mathrm{F}$ = ferrichromite; $\mathrm{M}=$ magnetite. A - From Michailidis (1995). B - From Hoffman \& Walker (1978).

4) (Golding \& Bayliss 1968, Paraskevopoulos \& Economou 1980)

COMPOSIÇÃO MINERALÓGICA Muitos autores afirmaram que a ferricromita não é uma espécie mineral devido ao fato de que ela representa um estágio fossilizado do reequilíbrio da cromita para magnetita (Bliss \& MacLean 1975, Hoffman \& Walker 1978, Mitra et al. 1992). No entanto, a única evidência concreta que sustenta essa afirmação vem do trabalho de Shen et al. (1988). Os autores estudaram ferricromitas do depósito de cromitito podiforme de HengChun, Taiwan, utilizando difratometria de elétrons e imagens de microscopia eletrônica de transmissão (STEM ). Identificaram a presença de um intercrescimento topotaxial de duas fases minerais como componentes da ferricromita: R3Ü4 e $\mathrm{RO}$, onde $\mathrm{R}=\mathrm{Fe}, \mathrm{Cr}, \mathrm{Mg}$ e $\mathrm{Al} . \mathrm{R}_{2} \mathrm{O}_{3}$ tem estrutura de espinélio e RO tem estrutura da halita. Localmente, foi identificada também a presença de uma fase $\mathrm{R}_{2} \mathrm{O}_{3}$, com estrutura do córindon. A granulometria dessas fases está entre poucas dezenas a poucas centenas de angstrons. Estes resultados indicam que a ferricromita não é um mineral mas um intercrescimento submicroscópico de fases óxidos.

É interessante observar que, quando se trata do intercrescimento apenas das fases $\mathrm{R}_{3} \mathrm{O}_{4}$ e $\mathrm{RO}$, ocorre um excesso de cátions bivalentes e um excesso no total de cátions em relação à proporção estequiométrica estabelecida para espinélios, chegando a 25,6 cátions totais para 32 oxigênios, caso a abundância de ambas seja igual. Se estiverem presentes somente as fases $\mathrm{R}_{3} \mathrm{O}_{4}$ e $\mathrm{R}_{2} \mathrm{O}_{3}$, e em iguais proporções, os cátions totais somarão 22,5 para uma normalização a 32 oxigênios e deverão conter um excesso de cátions trivalentes em relação aos bivalentes. A existência das três fases tenderia a "mascarar" o fato de que a ferricromita não é composta por uma única fase. Além disto, o cálculo estequiométrico do $\mathrm{Fe}_{+3}$, baseado na suposta estequiometria da ferricromita como espinélio, tende a eliminar o excesso ou a deficiência de cátions eventualmente presente.

GÊNESE O processo responsável pela formação da ferricromita é um tema ainda controverso. As principais hipóteses genéticas aventadas para a formação da ferricromita envolvem: A) processos magmáticos a tardi-magmáticos; B) serpentinização; C) metamorfismo regional de baixo grau de serpentinitos (serpentinização seguida de metamorfismo).

As hipóteses ligadas a processos magmáticos a tardi-magmáticos (A) foram questionadas por Bliss \& MacLean (1975). As evidências contrárias por eles levantadas: 1 - o trend de variação composicional da ferricromita difere daquele das cromitas ígneas; 2 - limite cromita-ferricromita: se a ferricromita fosse magmática os contornos do núcleo deveriam ser euédricos e não arredondados como observado e; 3 - ausência de inclusões entre a cromita e a ferricromita. Tais evidências refutam as hipóteses genéticas vinculadas a processos magmáticos.

A formação de ferricromita vinculada à serpentinização (B) é aventada por Beeson \& Jackson (1969), Ashley (1975), Paraskevopoulus \& Economou (1980) e Takla \& Noweir (1980). Alguns autores advogam a incapacidade da serpentinização de baixa temperatura, por si só, dar origem à ferricromita e invocam a necessidade de um metamorfismo ré- 
gional de baixo grau, posterior à serpentinização $(\mathrm{C})$, como condição necessária para tal (Bliss \& MacLean 1975, Takla 1982, Jan et al. 1985, Michailidis 1995). É importante frisar, no entanto, que muitas vezes há certa confusão nessa literatura com relação aos termos serpentinização, metassomatismo e metamorfismo regional, dificultando a compreensão dos processos geológicos contidos nas hipóteses aventadas pelos diversos autores para explicar a gênese da ferricromita. A serpentinização tanto pode ser interpretada como um processo metassomático ou como resultado de metamorfismo regional. O termo serpentinização (B) é entendido como um processo de baixa temperatura que produz lizardita/crisotila ( \pm brucita). A formação da antigorita \pm clorita é atribuída a metamorfismo regional de baixo grau superposto.

DISCUSSÃO Bliss \& MacLean (1975) associaram a formação da ferricromita à serpentinização mas afirmaram que este processo por si só não seria capaz de originar a ferri-

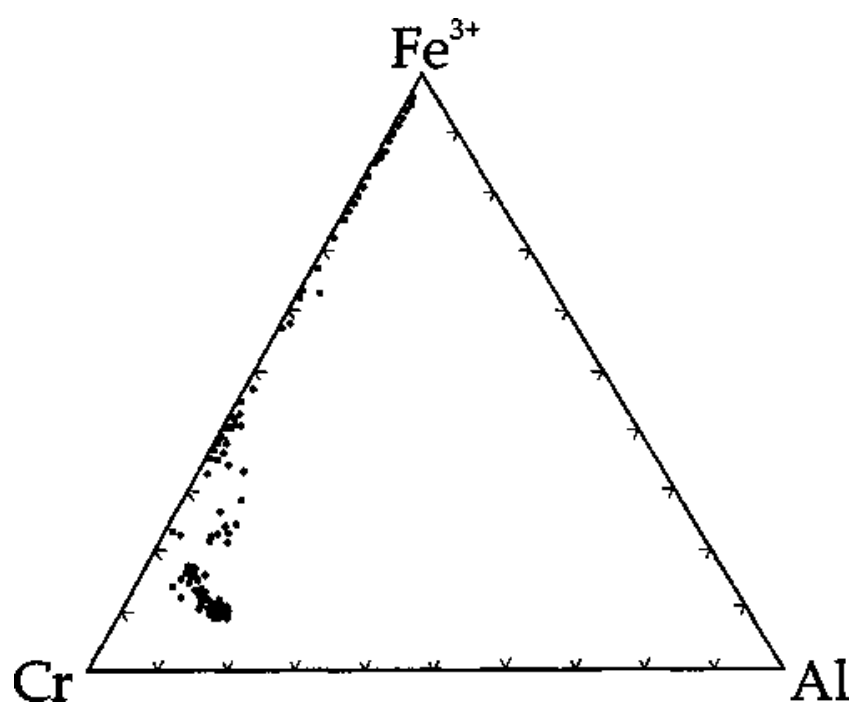

Figura 3 - Exemplo de variação composicional desde a cromita (pontos próximos ao vértice do $\mathrm{Cr}$ ), passando pela ferricromita e chegando até a magnetita (vértice $\mathrm{Fe}^{+3}$ ). Extraído de Michailidis (1995).

Figure 3 - Example of compositional variation from chromite (points near the $\mathrm{Cr}$ corner) through ferrichromite until magnetite $\left(\mathrm{Fe}^{+3}\right.$ corner). (From Michailidis 1995).

Tabela l-Exemplos de composições de cromita, ferricromita e magnetita

Table 1 - Examples of chromite, ferrichromite and magnetite compositions

\begin{tabular}{|c|c|c|c|c|c|c|c|c|c|}
\hline & IN & $2 \mathrm{~N}$ & $3 \mathrm{~N}$ & $\mathbf{I B i}$ & $2 \mathrm{Bi}$ & $3 \mathrm{Bi}$ & $\mathrm{LEc}$ & $2 \mathrm{Bc}$ & $3 \mathrm{Bc}$ \\
\hline $\mathrm{SrO}_{7}$ & 0,20 & & & 0.15 & & & 0.25 & & \\
\hline $\mathrm{TiO}_{2}$ & 0.52 & & 0.25 & 0.15 & 0.27 & 0.78 & 0.00 & 0,00 & 0.24 \\
\hline $\mathrm{Al}_{2} \mathrm{O}_{3}$ & 7.38 & 21.80 & 15.27 & 0.76 & 1.31 & 0.08 & 0.06 & 0.00 & 0.00 \\
\hline $\mathrm{C}_{2} \mathrm{O}_{3}$ & 56.86 & 46.00 & 48.32 & 53.73 & 37.50 & 28.65 & 7.17 & 3,43 & 3.87 \\
\hline $\mathrm{F} \in \mathrm{O}_{3}$ & 757 & 3.63 & 7.47 & 17.4 & 30.90 & 4158 & 62,80 & 66.190 & 65.44 \\
\hline$F \in O$ & 15.04 & 13.90 & 17.57 & 16.48 & 19.50 & 23.57 & 25.88 & 27,50 & 29.67 \\
\hline $\mathrm{MnO}$ & 1.00 & 0.69 & nd & 1.39 & 1.60 & nd & 0.33 & 0.15 & nd \\
\hline MyO & 11.20 & 1280 & 11.07 & 905 & 6,04 & 4.98 & 2.44 & 1.49 & 1.24 \\
\hline Nio & 0.19 & 0.10 & $0.0 \mathrm{t}$ & 0.13 & 0.39 & 0.45 & 1.49 & 1.14 & 0.56 \\
\hline Toplal & 99.96 & 98,96 & $100,00^{\circ}$ & 99.38 & 9751 & $99.99^{\circ}$ & 100,42 & 100.50 & 100,02 \\
\hline
\end{tabular}

cromita, baseados no fato do espinélio caracteristicamente formado ser a magnetita. Assim, propuseram o seguinte modelo: durante a serpentinização haveria o sobrecrescimento de magnetita neoformada na cromita e, posteriormente, num estágio de metamorfismo regional, no campo da antigorita, ocorreria uma tentativa de reequilíbrio entre a magnetita e a cromita em contato, originando um domínio intermediário de ferricromita.

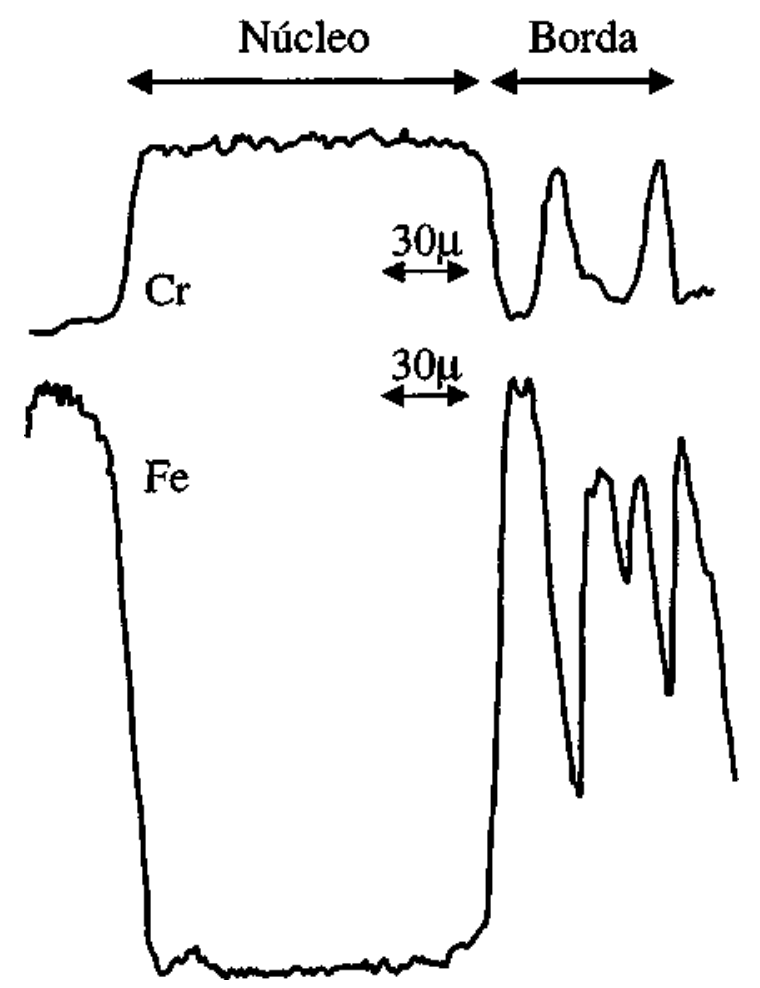

Figura 4 - Bandamento químico observado em domínios de ferricromita. Extraido de Paraskevopoulos \& Economou (1980).

Figure 4 - Chemical banding inside ferrichromite domains. From Paraskevopoulos \& Economou (1980).

A vinculação da ferricromita à serpentinização, e o fato da serpentinização por si só não formar ferricromita, passou a ser aceito por muitos dos autores que posteriormente se dedicaram ao assunto. No entanto, a hipótese de que a ferricromita resulta do reequilíbrio entre cromita e magnetita sobrecrescida foi rebatida. Os argumentos contrários baseiam-se no fato de que a saída do componente $\mathrm{MgAl}_{2} \mathrm{O}_{4}$ e a entrada de $\mathrm{Fe}_{3} \mathrm{O}_{4}$ no grão composto dá-se em relação ao exterior, ou seja, o componente $\mathrm{MgAl}_{2} \mathrm{O}_{4}$ irá participar da serpentina ou da clorita presentes no serpentinito, como atesta a presença de clorita e/ou Al-lizardita bordejando os grãos compostos. Além disto, existem os casos onde camadas de cromita cumulus sofrem reequilíbrio para ferricromita sem que haja qualquer evidência de ter existido uma precipitação de magnetita sobre a cromita (Figura 1A). Nesses casos, a clorita está sempre presente.

E fato que o processo de serpentinização forma magnetita pela liberação do ferro contido na olivina e/ou piroxênio. Esta magnetita pode formar trilhas no serpentinito, estar disseminada em finos cristais ou sobrecrescer grãos preexistentes de cromita (Figura 1 D). Por outro lado, observa-se, em alguns casos, que a borda de magnetita de grãos compostos com os contornos dos antigos cristais euédricos de cromita preservados, mostra feições de dissolução, tais como embainhamentos.

Wicks \& O'Hanley (1988) observaram que a magnetita pode ser reagente, produto ou agente passivo durante as 
transformações mineralógicas ocorridas em serpentinitos. A relação entre serpentinas e a variação modal de magnetita em serpentinitos foi estudada por O'Hanley \& Dyar (1993). Esses autores mostraram que a incorporação de $\mathrm{Fe}^{+3}$ no sítio tetraédrico da lizardita e antigorita, controlada principalmente pela $\mathrm{aSiO}_{2}$, tem grande influência sobre a precipitação ou dissolução da magnetita.

A necessidade da existência de metamorfismo regional posterior à serpentinização não é sustentada por argumentos convincentes. Por exemplo, recorre-se à presença de clorita no serpentinito ou ao fato de existir a substituição parcial de lizardita e crisotila por antigorita. Sabe-se que a antigorita tem seu campo de estabilidade estendendo-se até temperaturas mais altas do que a lizardita e a crisotila. No entanto, a lizardita é metaestável, sem um campo definido (Chernosky et al. 1988). Além disto, o limite superior de quebra da lizardita é diretamente proporcional à quantidade de $\mathrm{Al}$ que ela contem (Chernosky et al. 1988).

A presença de clorita nos serpentinitos está claramente associada à disponibilidade de $\mathrm{Al}$ e não com condições específicas de $\mathrm{P}$ e T. As auréolas de clorita em torno de grãos compostos por cromita/ferricromita/magnetita e a formação preferencial de clorita em vez de serpentina, em cromititos, (Beeson \& Jackson 1969, Fleet et al. 1993), atestam o controle preponderantemente químico (aA1) para a formação de clorita.

Há exemplos em que a cromita ocorre em serpentinitos sem a formação de ferricromita (Pinsent \& Hirst 1977). O que se observa neste caso é que a composição inicial da cromita é caracterizada por teores extremamente baixos de $\mathrm{Fe}_{2} \mathrm{O}_{3}$ e $\mathrm{Al}_{2} \mathrm{O}_{3}$. De acordo com os campos de solução sólida e desmistura calculados por Sack \& Ghiorso (1991) para $500^{\circ} \mathrm{C}$ (Figura 5), cromitas com composições próximas ao vértice $\mathrm{Cr}_{2} \mathrm{O}_{3}$ são estáveis nessas condições e não devem desenvolver mantos de ferricromita. Considerando que a serpentinização ocorre em temperaturas próximas, porém mais comumente abaixo de $500{ }^{\circ} \mathrm{C}$ (Chernosky et al. 1988), as lacunas de miscibilidade devem ser ainda maiores do que aquelas representadas na Figura 5.

Não vemos assim razões concretas para recorrer a um evento metamórfico regional posterior à serpentinização para o desenvolvimento de ferricromita a partir de cromita. Parece evidente que a serpentinização por si só é capaz de levar à formação de ferricromita, dependendo das condições em que este processo ocorre e da composição original da cromita.

As feições acima descritas têm implicações quanto ao significado e às interpretações petrogenéticas advindas das composições dos núcleos de cromitas em serpentinitos. Observou-se que: a) a zonação presente no núcleo de cromita pode ser semelhante àquela observada na ferricromita (Bliss \& MacLean 1975); b) pode existir um enriquecimento anômalo em Mn (Michaelides 1995) e/ou Zn (Bevan \& Mallinson 1980, Wylie et al. 1987,Liipo et al. 1995) no núcleo de cromita. O primeiro caso, quando presente, indica que esses núcleos de cromita já se apresentam em processo de transformação para ferricromita e que, portanto, não guardam mais sua composição química original. Neste caso, pode existir uma variação composicional entre diferentes núcleos de uma mesma amostra, resultante de diferentes graus de reequilíbrio destes núcleos.

Mn é claramente enriquecido na ferricromita em relação à cromita mostrando que este elemento está claramente associado ao reequilíbrio da cromita. Um eventual enriquecimento em Mn na cromita indicaria, portanto, que a composição atual do núcleo não é mais a original.

No caso das cromitas ricas em $\mathrm{ZnO}$, estudadas por Wylie et al (1987) (em mélanges de Sykesville, Maryland), pode-se observar que a zonação é caracterizada por um enriquecimento deste elemento da parte central do núcleo até o limite com a ferricromita. Neste caso, os autores concluíram que o $\mathrm{Zn}$ foi

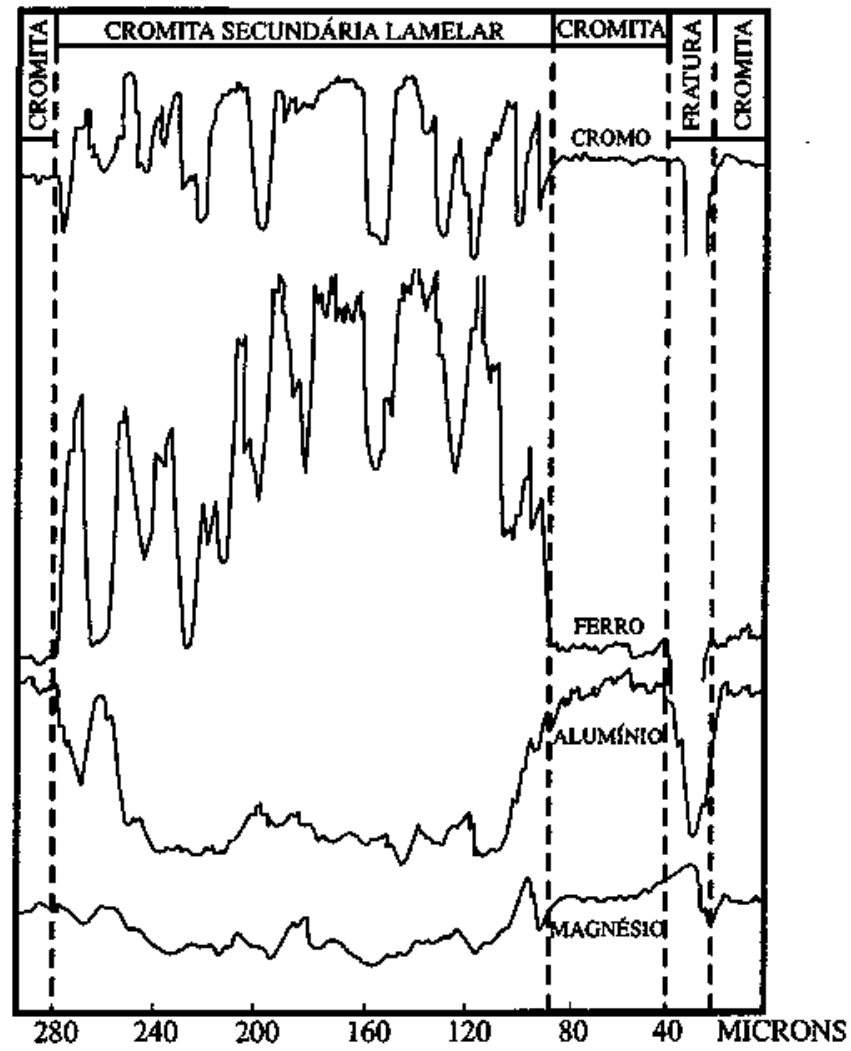

Figura 5 - Lacunas de miscibilidade para espinélios calculadas por Sack \& Ghiorso (1991).

Figure 5 - Spinel miscibility gaps as calculated by Sack and Ghiorso (1991).

incorporado na cromita durante o processo de formação da ferricromita que a bordeja, em múltiplos eventos hidrotermais que se estenderam desde a alteração hidrotermal de fundo oceânico até o metamorfismo regional.

Sugerimos então que cuidados especiais sejam tomados quanto ao significado que se pode depreender das composições químicas de cromita envolta por ferricromita. Somente quando trends composicionais tipicamente originais puderem ser observados pode-se utilizar tais dados para interpretações petrogenéticas mais amplas.

CONCLUSÕES a -Ferricromita não é um mineral, mas um agregado submicroscópico de fases oxido, de composição química variável entre os termos cromita e magnetita;

b - O padrão de variação química de grãos compostos por cromita, ferricromita e magnetita é constante nas ocorrências descritas, principalmente no que se refere aos elementos $\mathrm{Mg}$, $\mathrm{Al}, \mathrm{Fe}^{+2}, \mathrm{Fe}^{+3}, \mathrm{Mn}$ e Ni;

c - O processo responsável pela formação da ferricromita é a difusão no estado sólido de $\mathrm{Mg}$ e $\mathrm{Al}$, principalmente, e, muitas vezes, do $\mathrm{Cr}$, da fase oxido para as fases silicáticas circundantes, e de Fe para o interior da fase oxido. Os domínios de ferricromita enriquecem-se em Mn, enquanto as orlas de magnetita mostram enriquecimento em $\mathrm{Ni}$, este último proveniente da quebra da olivina durante a serpentinização;

d - Os núcleos de cromita aparentemente inalterados podem mostrar desvios da composição original, como, por exemplo, enriquecimento em Mn e/ou Zn. As zonações presentes na cromita podem não corresponder aos padrões atribuíveis aos processos originais, sendo, nestes casos, semelhantes ao pá- 
drão de variação química que caracteriza a formação da ferricromita;

e - O processo de formação dos grãos compostos de cromita -ferricromita - magnetita é essencialmente vinculado à serpentinização. Não há evidências da necessidade de um evento metamórfico regional superposto à serpentinização para que a ferricromita seja formada;

Agradecimentos Os autores agradecem o apoio financeiro da FAPESP e do CNPq.

\section{REFERÊNCIAS}

Ashley, P.M. 1975. Opaque mineral assemblage formed during serpentinization in the Coolac ultramafic belt, New South Wales. Jour. Geol. Soe. Austrália 22:91-102.

Beeson, M.H.; Jackson, E.D. 1969. Chemical composition of altered chromites from the Stillwater complex, Montana. Am. Min., 54:1084-1100.

Bevan, J.C; Mallinson, L.G. 1980. Zinc- and manganese-bearing chromites and associated grossular from Zimbabwe. Miner. Mag., 43:811-14.

Blais, S.; Auvray, B. 1990. Serpentinization in the Archean komatiitic rocks of the Kuhmo greenstonebelt, Eastern Finland. Can. Min. 28:55-66.

Bliss, N.W.; MacLean.W.H. 1975. The paragenesis of zoned chromite from central Manitoba. Geoch. Cosmoch. Acta, 39:973-990.

Candia, M.A.F. 1983. Petrologia dos Complexos Máficos-Ultrmáficos de Mangabal I e Mangabal H, Sanclerlândia, Gioás. Tese de Doutorado, Universidade de São Paulo, 400p.

Candia, M.A.F.; Gaspar, J.C. 1996a. Variações Composicionais em Cromo Espinélios de Rochas Ultrabásicas dos Complexos Mangabal I e II, Goiás. Anais do $39^{\circ}$ Congresso Brasileiro de Geologia, SBG, 6, 215-217.

Candia, M.A.F.; Gaspar, J.C. 1996b. Exsoluções em Cromo Espinélios. Revista Brasileira de Geociências, 26:87-92

Candia, M.A.F. ; Gaspar, J.C. 1997. Chromian Spinels in Metamorphosed Ultramafíc Rocks from Mangabal I and II Complexes, Goiás, Brazil. Mineralogy and Petrology, 60:27-40.

Chernosky Jr., J. V.; Berman, R.G.; Bryndzia, L.T. 1988. Stability, phase relations, and thermodynamic properties of chlorite and serpentine group minerais. In S.W. Bailey (ed)., Hydrous Phyllosilicates (Exclusive of Micos), Rev. Min., Min. Soe. Am., 19:295-346.

Fleet, M.E.; Angeli, N.; Pan, Y. 1993. Oriented chlorite lamellae in chromite from the Pedra Branca mafic-ultramafic complex. Ceará, Brazil. Am. Min 78:68-74.

Golding, H.G.; Bayliss, P. 1968. Altered chrome ores from the Coolac serpentine belt, New South Wales, Austrália. Am. Min., 53:162-183.

Haggerty, S.E. 1976. Opaque mineral oxides in terrestrial igneous rocks. In D Rumble, III (ed.); Oxide Minerais. Rev. Min., Min. Soe. Am., 3:101-300.

Hamlyn, P.R. 1975. Chromite alteration in the Panton Sill, east Kimberley region, Western Austrália. Min. Mag., 40:181-192.

Hoffman, M.A.; Walker, D. 1978. Textural and chemical variations of olivine and chrome spinel in the East Dover ultramafic bodies, South-Central Vermont. Geol. Soe. Am. Bull., 89:699-710.

Jan, M.Q.; Windley, B.F.; Khan, A. A. 1985. The Waziristan ophiolite, Pakistan: General geology and chemistry of chromite and associated phases. Econ. Geol., 80:294-306.

Khudeir, A.A.; Haddad, M.A.; Leake, B. 1992. Compositional variation in chromite from the Eastern Desert, Egipt. Min. Mag., 56:567-574.

Liipo, J.P.; Vuollo J.I.; Nykánen, V.M.; Pirainen, T.A. 1995. Zoned Zn-rich chromite from the Nãàtaniemi serpentinite massif, Kuhmo greenstone belt, Finland. Can. Min., 33:537-545.

Marques, J.C. 1996. Petrologia e metalogênese da seqüencia metaultramáfica da antiforme Canapé, suite metamórfica Porongos, Cachoeira do Sul-RS Dissertação de Mestrado, Universidade Federal do Rio Grande do Sul, 191p.

Michailidis, K.M. 1990. Zoned chromites with high Mn-contents in the Fe-Ni-Cr-laterite ore deposits from the Edessa Área in Northern Greece. Min.Dep., 25:190-197.
Michailidis, K.M. 1995. Compositional variation of zoned chromian spinels in serpentinites from the Kilkis Área, Northern Greece. Chem. Erde Geochem., 55:81-96.

Mitra, S.; Pai, T.; Maity, P.K.; Moon, H.S. 1992. Ferrichromit and its opto-chemical behaviour. Min. Jour., 16:173-186.

Nilson, A. A. 1981. The nature of the Americano do Brasil mafic-ultramafic complex and associated sulfide mineralization, Goiás, Brazil. Tese de Doutorado, Universidade de Western Ontario, London, Canadá. 460p.

O'Hanley, D.S.; Dyar, M.D. 1993. The composition of lizardite $1 T$ and the formation of magnetite in serpentinites. Am. Min., 78:391-404.

Onyeagocha, A.C. 1974. Alteration of chromite from the Twin Sisters dunite, Washington. Am. Min. 59, 608-612.

Paraskevopoulos, G.M.; Economou, M.I. 1980. Genesis of magnetite ore ocurrences by metasomatism of chromite ores in Greece. $N$. Jb. Miner. Abh., 140:29-53.

Pinsent, R.H.; Hirst, D.M. 1977. The metamorphism of the Blue River ultramafic body, Cassiar, British Columbia, Canada. Jour. Petrol., 18:567-594.

Sack, R. ${ }^{\circ}$ Ghiorso, M.S. 1991. An internally consistem model for the thermodynamic properties of $\mathrm{Fe}-\mathrm{Mg}$-titanomagnetite-aluminate spinels. Contr. Miner. Petrol., 106:474-505.

Shen, P.; Hwang. S.L.; Chu, H.T.; Jeng, R.C. 1988. STEM study of "Ferrichromit" from the Heng-Chum Chromitite. Am. Min., 73:383-388.

Spangenberg, K. 1943. Die Chromitlagerstatte von Tampadel in Zobten. Z. Prakt. Geol., 51:13-35.

Szabó, A.J. 1996. Petrologia da suite metaultramáfica da seqüencia vulcano-sedimentar Morro do Ferro na região de sul a oeste de Alpinópolis, MG (Domínio norte do complexo Campos Gerais). Tese de Doutorado, Universidade de São Paulo, 354p.

Takla, M.A. 1982. Chromites from the Bergen Ares ultramafics. NJb. Miner. Abi., 144:56-72.

Takla, M.A.; Noweir, A.M. 1980. Mineralogy and mineral chemistry of the ultramafíc mass of El-Rubshi, Eastern Desert, Egipt. NJb. Miner. Abh. 140:17-28.

Ulmer, G.C. 1974. Alteration of chromite during serpentinization in the Pennsylvania-Maryland District. Am. Miner., 59:1236-1241.

Wicks, F.J.; O'Hanley, D.S. 1988. Serpentine minerais: structures and petrology. In S.W. Bailey (ed) Hydrous Phyllosilicates (Exclusive of Micos), Rev. Min., Min. Soe. Am., 19:91-167.

Wylie, A.G.; Candeia, P.A.; Burke, T.M. 1987. Compositional zoning in unusual $\mathrm{Zn}$-rich chromite from the Sykesville District of Maryland and its bearing on the origin of "Ferrichromit". Am. Min., 72:413-422.

Manuscrito A925

Recebido em 15 de julho de 1997

Revisão dos autores em 20 de novembro de 1997 Revisão aceita em 25 de novembro de 1997 